\title{
A System Level Comparison of Drive Topologies for High Speed Electrical Machines
}

\author{
Filippo Savi, Davide Barater \\ Departement of engineering and architecture \\ University of Parma, Parma, Italy \\ Email: filippo.savi2@ studenti.unipr.it
}

\author{
Mauro Di Nardo, Michele Degano, Chris Gerada \\ Departement of electric and electronic engineering \\ University of Nottingham, Nottingham, UK \\ Email: mauro.dinardo@nottingham.ac.uk
}

\begin{abstract}
This paper presents a comprehensive comparative study among five different converter topologies all designed to drive a $8.5 \mathrm{~kW}-120 \mathrm{krpm}$ surface permanent magnet synchronous machine. The study aims at comparing the considered systems in terms of converter complexity, control complexity and overall efficiency. The assessment of the subsystems' efficiencies is based on a set of decoupled converter-electrical machine simulations. First the designed converters are simulated in Matlab-Simulink environment in order to estimate the converter losses and the current waveforms. Then the latter are used to supply the FE model of the electrical machine so to estimate all the loss components present in the real scenario. The results of the carried out study gives a wide understanding of the interaction between the two subsystems and some general design considerations needed to select the converter topology.
\end{abstract}

\section{INTRODUCTION}

In recent years there has been a considerable interest towards the progressive electrification of aircraft. This concept, generically called More Electric Aircrafts (MEA) [1], [2], consists in the replacement of hydraulic, pneumatic and mechanical systems with electrical ones. The substitution of the mechanical actuators used for the primary and secondary flight control and the systems needed to drive the fuel pumps [3] with electro-mechanical actuators involves the redefinition of the power distribution systems and a considerable increase of the overall electrical power demand of the aircraft. With the aim of reducing the generation system complexity and increase the reliability and the efficiency, several studies have focused on the integration of the electrical generator directly inside the main gas turbine engine. This concept is named More Electric Engine (MEE) and it could, partially or totally, eliminate the constant velocity gearbox (present in the commercial aircraft to drive at constant speed the electrical machine from the main engine shaft). In particular, the electrical generator can be integrated within the main engine in different positions, on the low or high pressure shaft. The latter approach leads to higher performances, in the face of increased complexity. The interest for high speed electrical machines is not exclusive of the aviation sector [4], [5]. In fact, several other industrial applications can benefit from the introduction of high speed electrical machine within the system such as oil and gas compressors, spindle, turbocharger, micro-turbines, turbo-molecular pumps, etc. Such a machine can in fact be directly coupled to the load, eliminating the inefficiencies of gear/belt/turbine driven transmission systems This increased interest in applications of high speed electrical machine has been only possible due to improvement in methodologies of manufacturing, bearing technologies, and the availability of high specifications materials and new power electronic devices [6]. In spite of the above mentioned advantages, as the speed increases, several limitations related to mechanical, thermal, control system and power electronics issues arise. Regarding the electrical machine, the rotor structural integrity and the high frequency additional losses both in the laminations and conductors are the main challenges that the designer has to face. Regarding the power electronic, the main challenge is the difficulty of synthesizing a multiple phase current system with a high fundamental frequency with the minimum harmonic content. The introduction of wide-bandgap materials ( $\mathrm{SiC}$ and $\mathrm{GaN}$ ) and their use in power electronic devices has allowed coping easier these requirements, being higher the maximum switching frequency and/or lower the switching losses compared to standard devices (Si). The higher switching frequency allows also a better level of controllability and reduce, or completely eliminate, the dimensions of the output and input filters traditionally used to respectively minimize the total harmonic distortion of the input voltage and the output current ripple. The correct design of a high speed drive must not overlook the effect that the converter has on the performance of the whole system. In fact, on one hand, to minimize the converter rating (and so its cost), the efficiency and the power factor of the machine needs to be as high as possible. On the other hand, the machine efficiency is affected by the converter topology and its output current quality. Several works have independently addressed the problem of selecting the most suitable electrical machine [7] or converter topology for a certain high speed application. However, considering independently the converter [8], [9] and the electrical machine [10], can lead to a net decrease in the overall efficiency of the system. Few studies have instead faced the problem considering the effect of the converter choice on the electrical machine and vice-versa. By doing so, i.e. carefully designing the overall electrical drive not placing too much emphasis on the component level, it is possible to achieve the highest efficiency.

The next section gives a brief description of the electrical machine, section III outlines the converter layouts; section IV details the peculiarities of both power electronic and electrical machine simulations while the results of the carried out study are reported in section $\mathrm{V}$. 


\section{High Speed Electrical Machines}

\section{A. Overview}

Three types of electrical machines have been proposed in literature and adopted in industry for high speed applications: induction motors (IM), switched reluctance (SR) and surface mounted permanent magnet synchronous machines (SPMSM). Each of them has its own advantages and disadvantages, and their selection strongly depends on the considered application [4]. The high robustness of the rotor structures of IMs, laminated or solid ones, makes this machine topology very suitable for high speed applications. The absence of permanent magnets (PMs) and retaining sleeves inherently removes any demagnetization issues and simplifies the rotor construction leading to a really cost-effective solution. The high rotor losses, the low power factor and efficiency constitute their main disadvantages. Consequently IMs are less favourable for application where the efficiency and the power factor are the most important requirements. SR machines are another valid alternative used in HS applications due to their intrinsically simple rotor construction, lack of active components and containment system on the rotor. The ability to operate in really harsh environments, and its inherent fault tolerant capability are the most important advantages of this machine topology along with its robust rotor structure. The need of magnetizing current obviously deteriorates both efficiency and power factor. In addition, due to the double salient structure, windage losses tends to become higher respect to the alternative topologies. SPMSMs exhibit the best performance in terms of torque density, power factor and efficiency. These advantages are strictly related to the use of high energy density permanent magnet (based on rare-earth materials), and the consequent lack of magnetizing current and low rotor joule losses. Its major challenge is associated with the design and the manufacturing of the magnets retention system required to guarantee the rotor integrity. Both the PMs and the retaining sleeve are subject to additional high frequency losses; the latter need to be minimized during the design stage in order to respect the thermal limits of the winding and PMs. In fact, both remanent flux density and coercivity of the rare-earth based PMs decrease as the temperature increases, deteriorating the ability to produce magnetic flux and increasing the risk of demagnetization.

\section{B. Developed SPMSM design}

Being the power density, the efficiency and the power factor, the most important requirements of the considered application, a SPMSM has been designed to deliver $8.5 \mathrm{~kW}$ at $120 \mathrm{krpm}$. Table I reports the most salient machine specifications and geometrical parameters. Several slot/pole combinations and winding configurations have been compared in terms of efficiency, distribution of the losses and thermal behaviour for the considered requirements as reported in [11]. The distributed winding configuration, featuring 24 slots with 4 poles, allows achieving the best thermal-wise distribution of the losses, i.e. a design with the lowest rotor and the stator iron losses higher than the copper losses. Although this configuration does not provide the highest efficiency, the losses are located

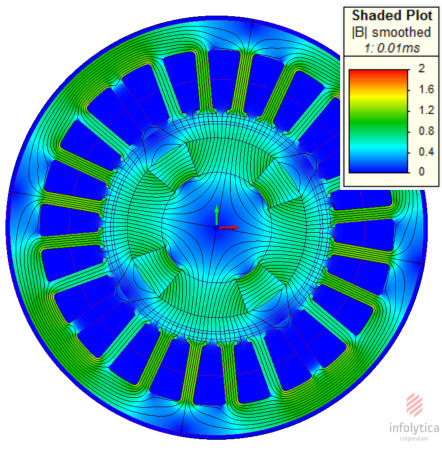

Fig. 1: Flux density distribution at no load

where they are easier to extract, so allowing a simpler thermal management. Following the above mentioned trade-off study, which included a mechanical analysis aimed at selecting the sleeve thickness (made of Inconel alloy) and to calculate the critical frequency of the rotor system, a quasi-Halback SmCo magnets array has been chosen. This choice has been made in order to increase the fundamental air-gap flux density, and so the power density, and to reduce the manufacturing complexity of the rotor assembly. Moreover, with the aim of decreasing the stator iron losses, a 0.007 in thick lamination material is selected having a low specific iron losses and the machine is designed to work with a low iron flux densities as depicted in Fig. 1.

As above mentioned, the developed design has aimed at minimizing the rotor losses, i.e. the losses due to the eddy currents induced in rotor conductive part by airgap space harmonic fields, rotating asynchronously with respect to the rotor [12]. These harmonics are the consequence of the geometric arrangement of the conductors along the stator circumference, the slotting effect and the stator current distortion. The selected slot/pole combination allows reducing this loss component disregarding the stator current distortion, therefore assuming an ideal sinusoidal waveform. The performance alteration of the designed machine due to the current distortion will be analyzed in detail in Section IV and V considering 5 different converter topologies.

\section{Converter ArChitectures Overview}

The most widely used electric drive architecture is based on the Voltage Source Inverter (from now on called VSI) shown in Fig. 2. Its well-known structure consists in a capacitively filtered DC-link feeding a classic three phase full bridge;

TABLE I: Electrical machine specifications and parameters

\begin{tabular}{|c|c|c|}
\hline Parameter & Value & Unit \\
\hline Rated power & 8.5 & $\mathrm{~kW}$ \\
\hline Maximum speed & 120 & $\mathrm{krpm}$ \\
\hline Stator slots & 24 & - \\
\hline Pole pairs & 2 & - \\
\hline Stator Diameter & 60 & $\mathrm{~mm}$ \\
\hline Split ratio & 0.54 & - \\
\hline Stack length & 30 & $\mathrm{~mm}$ \\
\hline Airgap length & 1.5 & $\mathrm{~mm}$ \\
\hline Coolant flow rate (water) & 15 & $1 / \mathrm{m}$ \\
\hline
\end{tabular}


an inductive filter might be used to reduce the output's harmonic content. The main benefits of this topology are its low hardware and control complexities. In fact, hardwarewise, it requires only a fixed dc-link voltage, so without the need of a pre-regulation stage (generally required for other topologies), while software-wise, the control algorithms are widely available in technical literature.

Another converter architecture is the Current Source inverter (also known as CSI), shown in Fig. 3 where a constant current, generated by a switching pre-regulator and filtered with a high value DC-Link inductor, is periodically switched across two of the load's phases. This topology requires a capacitive output filter to smooth voltage spikes caused by the motor's inductance that would otherwise quickly destroy the active devices. One of the main benefit of this topology is the easy integration within electric drive systems as it greatly simplifies the currents control, that could be in some cases implemented with simple feed-forward schemes. Another advantage is the inherent protection against over-current and short circuits due to the presence of the large inductor in series with the main current path of the converter. This constitutes also one of the main drawbacks, i.e. the poor dynamic response to load's torque variations. The need of a unidirectional current flow on the inverter legs dictates the use of diodes in series with the main active components when they are not naturally reverse blocking. Consequently, this converter is generally used to drive very high power machines, in thyristor based converter. The choice of control modulation schemes for this architecture is quite limited.

If switching losses are the prevalent portion of loss, as it is typically the case for an inverter working at very high speeds, the two previous architectures can be modified by adding a pre-regulation stage that controls the DC-link's level and consequently the output's amplitude. The output frequency is then controlled by the actual bridge itself. This allows reducing the switching frequency (which will be equal to the output's

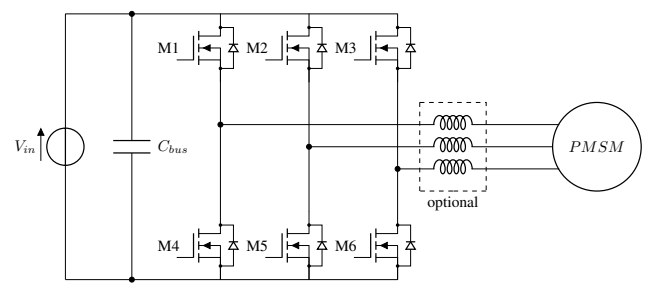

Fig. 2: Voltage Source Inverter

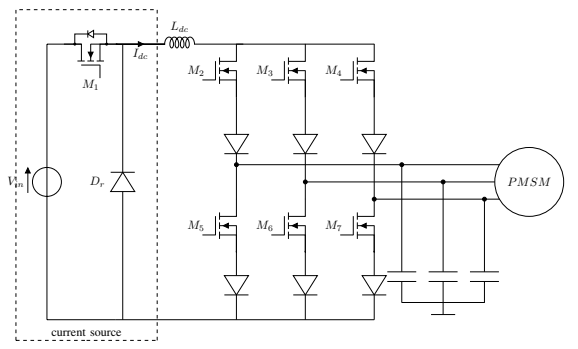

Fig. 3: Current Source Inverter

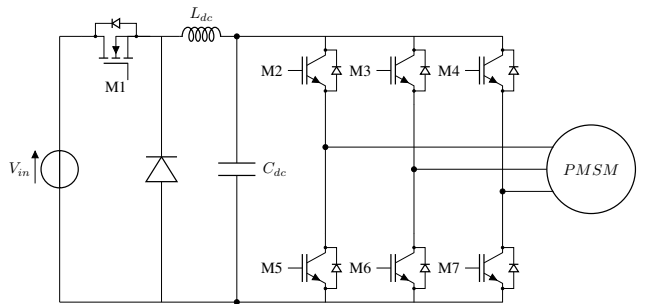

Fig. 4: Voltage Source Inverter with dc link pre-regulator

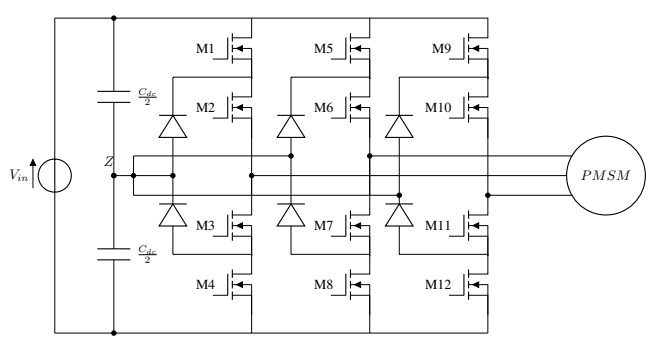

Fig. 5: Three level Neutral Point Clamped Inverter

one), thus reducing the overall switching losses; this mode of operation is generally known as six step modulation. The current source inverter can naturally work in this fashion with the appropriate switching times and sequences, while the VSI needs to be modified to include a buck regulator on the input side, with the relative LC filter, as shown in Fig. 4

The last considered architecture which can be used in high speed application is based on the Neutral Point Clamped (NPC) multilevel inverter. In this case, the output's waveform is synthesized from three discrete voltage levels (zero, full and half the DC voltage) in case of a three level inverter. In order to generate the additional level, a split series capacitor arrangement is used, as shown in Fig. 5; the resulting neutral point must be carefully controlled to avoid output's quality degradation. In this topology, the devices of a classic full bridge are substituted with a series of two different transistor and a diode, with which the load is clamped to the aforementioned neutral point, when the two middle devices are on; in the other two operating mode, the behaviour of this converter is equal to the classic Voltage source inverter. The main advantage of this topology is the capability to operate at higher output switching frequency without increasing the single devices operating frequency and so potentially reducing the dynamic losses. The configuration with two transistors in series allows also the use of devices with a lower breakdown voltage rating, lowering the losses even further. The two drawbacks of this topology are the high number of active devices required and the complexity of the control algorithm. Indeed, 12 transistors and 6 diodes for a three level NPC converter are needed against only 6 transistors for a simple voltage source inverter. The higher complexity of the modulation algorithm is mainly due to the increased number of possible switching states and the need of implementing a strategy to balance the neutral point. 
TABLE II: Devices used for each converter topology

\begin{tabular}{|c|c|c|}
\hline Topology & Bridge Devices & Preregulator Devices \\
\hline VSI & SiC NMOS & - \\
\hline Six Step VSI & Si IGBT & SiC NMOS \\
\hline NPC & GaN HEMT & - \\
\hline CSI & SiC NMOS & - \\
\hline Six Step CSI & Si IGBT & SiC NMOS \\
\hline
\end{tabular}

\section{Simulations Settings}

\section{A. Drive Simulations}

With the aim of comparing the performances of the various drive topologies presented in the previous section, several electro-thermal simulations in Matlab-Simulink environment have been carried out, at the rated current and the maximum speed. The hardware design of the various converters follows closely the previously discussed considerations. The only difference is given by the absence of the output filter in the voltage source based topologies; the introduction of such component would negatively affect the drive's dynamic performances, in particular the output current maximum rate of change and so the load's variation response time. In order to increase the efficiency, devices using wide band-gap materials have been chosen; in particular Silicon Carbide MOSFET's (ST microelectronics SCT20N120) have been selected for all the converter except for the multilevel NPC inverter, where the lower breakdown voltage requirement has made the usage of gallium nitride devices (GaN systems TPH3208P). As reported in Table II, which summarizes the device technologies used for each converter, both current source and voltage source topologies controlled in six step operation make use of $\mathrm{Si}$ devices for the main bridge and $\mathrm{SiC}$ devices for the preregulator circuit. To be able to capture both thermal and electric effects with a single simulation of reasonable running length, a piecewise-linear approach has been used. After the initial transient, all the current and voltage waveforms along the power losses have been recorded. Once the simulation reached the steady state, the load current's harmonic profile has been calculated by mean of a fast Fourier transform (using a hanning window to lessen the border discontinuity effects). These current waveforms are then used to evaluate the losses of the machine in realistic operating conditions as discussed in the next sub-section.

\section{B. Electric Machine FE Simulations}

The challenge of evaluating the electromagnetic losses of a high speed permanent magnet machine lies in the difficulties of calculating 1) the copper losses due to the non-uniform current density distribution within the slot, 2) the iron losses in the laminated part of the machine and 3) the eddy current losses in the solid component (i.e. sleeve and magnets). The calculation of the skin and proximity component of the AC copper losses can be addressed by analytical means. The determination of the circulating current component of the AC copper losses (arising in case the single conductors are made of more parallel strands and the conductors are not transposed along the axial length of the machine) requires more effort as this phenomena is strictly related to the real positions of the strands inside the slot. Several works have been recently presented in literature which try to calculate the circulating currents both by analytical method (essentially based on circuital approach) [13] and brute-force FE study [14]. In the following, a simple FE simulation of the final winding arrangement has been carried out in order to estimate the dependence of the resistance factor $\left(R_{a c} / R_{d c}\right)$ with the frequency in the ideal supply condition. The copper loss terms due to a certain time harmonic has been then simply calculated from the relative dc copper loss and the resistance factor. The stator iron losses have been calculated by using a modified Steinmetz model fitting the manufacturer loss data. With the aim of be able to evaluate the influence of the current harmonics on the machine performance, it is clear that the number of steps used to simulate one electrical period depends on the maximum harmonic order to be considered. For all the considered converters, the considered maximum current harmonic order is the 50th. The precise computation of the eddy-current losses in the permanent magnets and the retaining sleeve would require a 3D FEA with a remarkable fine mesh in the regions where the eddy current arise. Several analytical and semi-analytical methods have been lately introduced in the literature in order to avoid such high computational expensive simulation. In this work, the approach proposed in [15] has been used to determine the eddy current losses in the solid parts of the rotor. This approach consists in performing a 2D FE simulation with an adjusted value of the permanent magnet resistivity in order to consider its temperature variation and a correction factor accounting for the three-dimensional nature of the problem.

\section{Simulation Results}

\section{A. Drive Simulations}

1) Power losses: The power losses in the active devices for various architectures are shown in Fig. 6. The use of a Six Step Voltage Source inverter therefore bears no advantages over the traditional one. The current source inverter shows very high losses due to the fixed DC-link current always circulating in two of the output bridge's devices. In this case albeit the bulk of the losses is composed by conduction losses the Six step modulation cuts the overall figure by virtue of the pre-regulator dc-link current's amplitude control function. The best architecture in this regard is the multilevel neutral point clamped inverter, even though the architecture's conduction losses will raise linearly with the number of levels due to the higher number of conducting devices in series, the lower breakdown voltage enables the use of lower rated devices, that generally have a much lower $R_{d s_{o} n}$, the net effect is a lower overall losses.

2) Output Power's Quality: From the analysis of load currents' spectrum, as shown in figure 7, several remarks can be made: the Current Source topologies shows a high distortion at a third of the nominal frequency because of an undamped resonance in the LCL network constituted by DCLink and load inductances together with the filter's capacitors. Everywhere else in the operating range this converter behaves as expected in particular, it has a very low distortion at nominal 


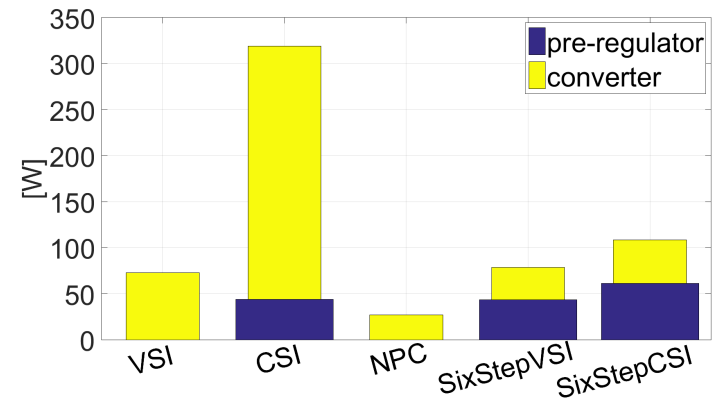

Fig. 6: Simulated power losses in active devices

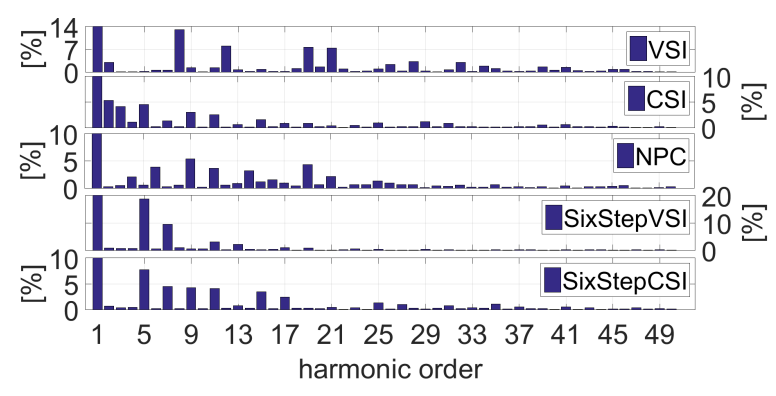

Fig. 7: Simulated output's current spectrum

frequency that gradually worsens due to the output filter's loss of effectiveness against the lower harmonics. The Six step inverters have as expected a higher distortion since the lower output's frequency. In voltage source and multilevel NPC inverters, whose distortion is roughly comparable, there is, contrary to expectations, a mildly decreasing distortion as the output frequency drops, this is due to a combinations of two different factors, the first one is the lack of an output filter whose effectiveness at filtering unwanted harmonics drops significantly at lower frequencies, this enables the second factor to come into play, namely the first few undesired harmonics, as the fundamental frequency goes down, will enter into the pass band of the current's PID regulator and will be rejected. This effect whilst always present can be observed only on inverters with unfiltered output, since the output's filter's attenuation roll-off as frequency increases is much greater and completely masks this effect.

\section{B. Electric Machine Simulations}

The machine losses FE-evaluated with the methods presented in section IV-B are reported in Fig. 8. This picture depicts the four main components of the machine losses (i.e. stator iron losses, copper losses and eddy current losses in the sleeve and in the magnets) for all the five converter topologies considered and for the ideal supply condition. The variation of each loss component with respect to the ideal supply condition is shown in Fig. 9. Clearly the stator iron and copper losses constitute the main portion of the global losses since the eddy current losses on the rotor components have been properly minimized during the design stage of the machine. When the machine is supplied with any of the considered converters, the eddy current losses in the magnets and in the sleeve drastically increase respectively of an order about $10^{4}$ and $10^{3} \%$, while the stator iron and copper losses increments are in the order of $10 \%$. Fig. 10 reports the total machine loss and the current THD variations with respect to the ideal supply condition. Comparing the machine losses variations due to the different current harmonics produced by the 5 converters, the following considerations can be drawn.

- The copper losses obviously follow the trend on the current THD while the other loss components do not follow such trend since they depends on the properties of the harmonic spectrum.

- The highest machine loss variation, so the worst converter architecture, is the VSI. In fact, it provides the highest variation of all the loss components, being the stator iron losses the biggest one in absolute terms and the magnet losses in relative term.

- Although the VSI and the six step VSI provide a current waveform having a similar THD, the second one performs better loss-wise because the current harmonics are located in the lower frequency range leading to a conspicuous decrease of the iron losses and rotor losses.

- The best converter topology is the CSI although the NPC architecture provides the lowest current THD. This is due to the position of the current harmonics in the spectrum, the CSI provide a current waveform whose distortion is in the low frequency range leading to a lower iron losses respect to the NPC.

\section{Overall Performance}

The total machine and converter losses and the global efficiencies of the 5 considered converters are shown in Fig. 11. The NPC provides the best total efficiency while the CSI is the worst among the studied architectures although it provides the

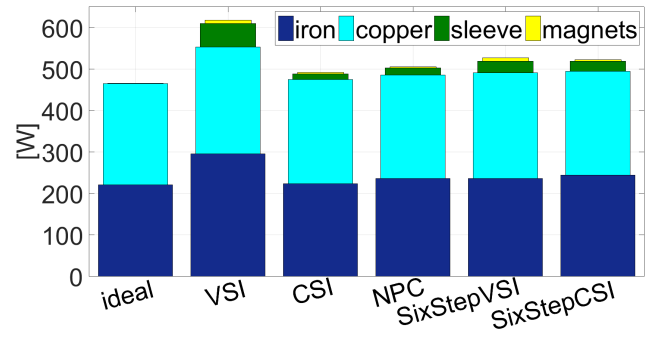

Fig. 8: Power losses of the electrical machine

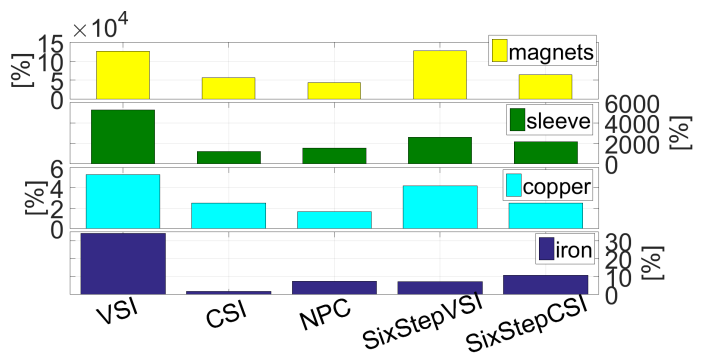

Fig. 9: Power losses variations (respect to the ideal supply condition) of the electrical machine 


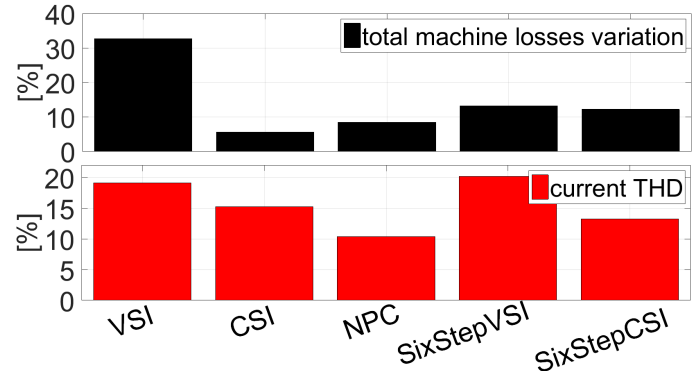

Fig. 10: Total power losses variation (respect to the ideal supply condition) of the electrical machine and current THD

lowest machine losses. It is worth to notice that both six step current and voltage source inverters lead to a better efficiency respect to the VSI due to the lower machine losses (caused by a current distortion made of low frequency harmonics).

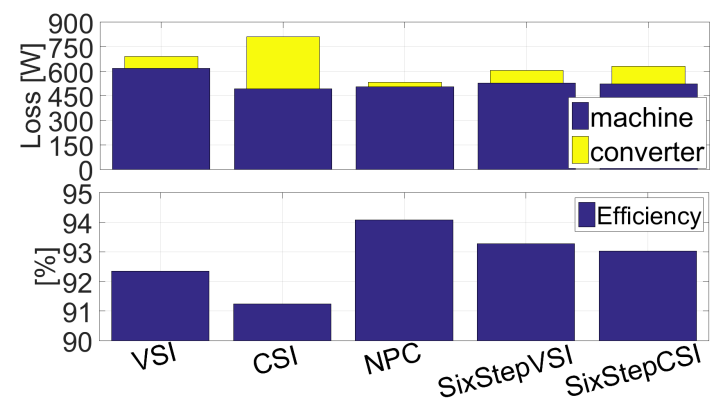

Fig. 11: Total Losses and efficiency comparison amongst all the studied architectures

\section{CONCLUSION}

A comprehensive comparison among the most common converter topologies used to drive high speed electrical machine has been presented. By coupling Power electronics and finite elements electromagnetic machine's simulations, we have been able to identify in the neutral point clamped multilevel inverter; the most favorable converter architecture to drive high speed permanent magnet synchronous machines with the highest possible system efficiency.

\section{REFERENCES}

[1] R. I. Jones, "The more electric aircraft: the past and the future?" in IEE Colloquium on Electrical Machines and Systems for the More Electric Aircraft (Ref. No. 1999/180), 1999, pp. 1/1-1/4.

[2] J. A. Rosero, J. A. Ortega, E. Aldabas, and L. Romeral, "Moving towards a more electric aircraft," IEEE Aerospace and Electronic Systems Magazine, vol. 22, no. 3, pp. 3-9, March 2007.

[3] A. Boglietti, A. Cavagnino, A. Tenconi, S. Vaschetto, and P. di Torino, "The safety critical electric machines and drives in the more electric aircraft: A survey," in 2009 35th Annual Conference of IEEE Industrial Electronics, Nov 2009, pp. 2587-2594.

[4] R. Abebe, M. D. Nardo, D. Gerada, G. L. Calzo, L. Papini, and C. Gerada, "High speed drives review: Machines, converters and applications," in IECON 2016 - 42nd Annual Conference of the IEEE Industrial Electronics Society, Oct 2016, pp. 1675-1679.

[5] D. Gerada, A. Mebarki, N. L. Brown, C. Gerada, A. Cavagnino, and A. Boglietti, "High-speed electrical machines: Technologies, trends, and developments," IEEE Transactions on Industrial Electronics, vol. 61, no. 6, pp. 2946-2959, June 2014.

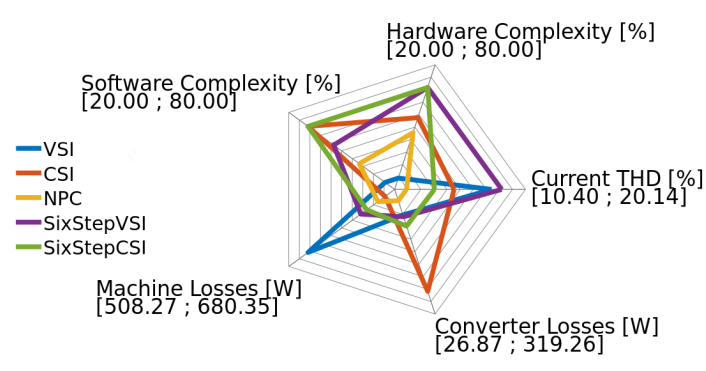

Fig. 12: Visual comparison amongst all the studied architectures

[6] J. B. Bartolo, H. Zhang, D. Gerada, L. D. Lillo, and C. Gerada, "High speed electrical generators, application, materials and design," in 2013 IEEE Workshop on Electrical Machines Design, Control and Diagnosis (WEMDCD), March 2013, pp. 47-59.

[7] G. L. Calzo, P. Zanchetta, C. Gerada, A. Gaeta, and F. Crescimbini, "Converter topologies comparison for more electric aircrafts high speed starter/generator application," in 2015 IEEE Energy Conversion Congress and Exposition (ECCE), Sept 2015, pp. 3659-3666.

[8] G. J. Su and P. Ning, "Loss modeling and comparison of vsi and rb-igbt based csi in traction drive applications," in 2013 IEEE Transportation Electrification Conference and Expo (ITEC), June 2013, pp. 1-7.

[9] L. Clotea and A. Forcos, "Power losses evaluation of two and three-level npc inverters considering drive applications," in 2012 13th International Conference on Optimization of Electrical and Electronic Equipment (OPTIM), May 2012, pp. 929-934.

[10] R. Andersson and A. Reinap, "Loss mapping of an insert permanent magnets synchronous machine for parallel hybrid electric heavy vehicles," in 2016 XXII International Conference on Electrical Machines (ICEM), Sept 2016, pp. 1847-1853.

[11] D. Borg-Bartolo, D. Gerada, C. Micallef, A. Mebarki, N. L. Brown, and C. Gerada, "Thermal modelling and selection of a high speed permanent magnet surface mount electrical machine," in 6th IET International Conference on Power Electronics, Machines and Drives (PEMD 2012), March 2012, pp. 1-6.

[12] F. Luise, A. Tessarolo, F. Agnolet, and M. Mezzarobba, "Use of time-harmonic fe analysis to compute rotor eddy-current losses in synchronous machines subject to distorted stator currents," in 2012 XXth International Conference on Electrical Machines, Sept 2012, pp. 15031509.

[13] A. Lehikoinen, N. Chiodetto, E. Lantto, A. Arkkio, and A. Belahcen, "Monte carlo analysis of circulating currents in random-wound electrical machines," IEEE Transactions on Magnetics, vol. 52, no. 8, pp. 1-12, Aug 2016.

[14] M. van der Geest, H. Polinder, J. A. Ferreira, and D. Zeilstra, "Current sharing analysis of parallel strands in low-voltage high-speed machines," IEEE Transactions on Industrial Electronics, vol. 61, no. 6, pp. 30643070, June 2014.

[15] S. Ruoho, T. Santa-Nokki, J. Kolehmainen, and A. Arkkio, "Modeling magnet length in 2-d finite-element analysis of electric machines," IEEE Transactions on Magnetics, vol. 45, no. 8, pp. 3114-3120, Aug 2009. 